Original Article

\title{
Functional difference between the proximal and distal compartments of the semitendinosus muscle
}

\author{
Koji Watanabe, $\mathrm{PT}^{1)^{*}}$, Satoru Otsuki, $\mathrm{PT}^{1)}$, Takushi Hisa, $\mathrm{PT}^{1)}$, Masanori Nagaoka, MD, PhD ${ }^{2)}$ \\ 1) Juntendo Tokyo Koto Geriatric Medical Center: 3-3-20 Shinsuna, Koto-ku, Tokyo 136-0075, Japan \\ 2) Department of Rehabilitation Medicine, Juntendo University Graduate School, Japan
}

\begin{abstract}
Purpose] The tendinous inscription divides the semitendinosus muscle into the proximal and distal compartments. It was hypothesized that there are functional differences between those compartments. [Subjects and Methods] Seven adult males performed knee flexion and hip extension in the prone position. An ultrasound device measured the decrease in the length of muscle fibers in the two compartments during these movements. The knee and hip joint angles were concurrently measured using a video camera. Pearson's correlation coefficients were calculated between the decrease in muscle fiber length in each compartment and joint angle. [Results] During knee flexion, decreased muscle fiber length was significantly correlated with increased knee flexion angle. During hip extension, there were no significant correlations for either compartment. Only the decrease in muscle fiber length in the distal compartment during hip extension tended to be negative; the other decreases in muscle fiber length tended to be positive. [Conclusion] Correlations did not reveal any functional differences. However, only the distal compartment elongated during hip extension. This result might show a functional difference and could be applied in clinical contexts during hip extension.

Key words: Semitendinosus muscle, Tendinous inscription, Skeletal muscle function
\end{abstract}

(This article was submitted Nov. 20, 2015, and was accepted Feb. 2, 2016)

\section{INTRODUCTION}

Muscle contraction produces force and change in the muscle's length, i.e., velocity. These parameters will change depending on the muscle's structure ${ }^{1)}$. Skeletal muscles are primarily classified into parallel muscles and pennate muscles, based on the muscle's form and structure. In parallel muscles, the shortening velocity is faster and the range of motion is larger because the muscle fibers are arranged longitudinally and directly reflect mechanical shortening. In contrast, in pennate muscles, the shortening velocity is slower and the range of motion is shorter because muscle fibers are arranged diagonally with a certain pennate angle. Pennate muscles exert stronger force because they comprise a greater number of muscle fibers, with a larger cross-sectional area. Generally, parallel muscles are located at joints, such as the elbow joint, and require exercise to change the joint angle significantly. Conversely, many pennate muscles are located in the lower extremities and counteract the effects of gravity ${ }^{2,3)}$. Therefore, the forms of skeletal muscles closely relate to their function.

The semitendinosus muscle (ST) composes a part of the hamstrings and has a characteristic form (Fig. 1). The ST has a tendinous inscription (TI) in the center of the muscle belly, and its muscle fibers are divided into a proximal compartment (PC) and a distal compartment (DC). The PC of the ST is a parallel muscle, but the DC has a pennate structure with an average $12.9^{\circ}$ pennate angle ${ }^{4}$. In other words, the ST comprises both parallel and pennate muscles dependent on the existence of the TI. Functional differences can therefore be assumed between the two compartments, because each has a unique innervation ${ }^{5,6)}$.

Reportedly, the ST is susceptible to injury in the DC on the sports field ${ }^{7}$. It is believed that the muscle fibers are particularly easily injured during eccentric contraction because they cannot withstand elongation due to the load ${ }^{3,8)}$. For example,

\footnotetext{
*Corresponding author. Koji Watanabe (E-mail: kjwatana@juntendo.ac.jp)

(C)2016 The Society of Physical Therapy Science. Published by IPEC Inc.

This is an open-access article distributed under the terms of the Creative Commons Attribution Non-Commercial No Derivatives (by-nc-nd) License $<$ http://creativecommons.org/licenses/by-nc-nd/4.0/>.
} 


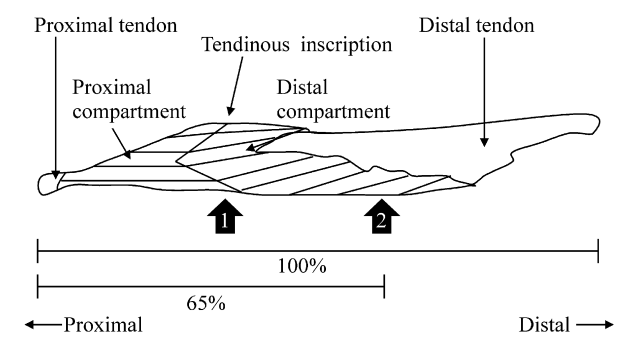

Fig. 1. Structure of the semitendinosus muscle

The line of tendinous inscription (arrow 1) lies at the center of the muscle belly. The distal tendon is longer than the proximal tendon. The proximal compartment has the structure of a parallel muscle, whereas the distal compartment is considered a pennate muscle because it has a pennate angle (arrow 2 ; recorded at $65 \%$ from the proximal tendon).

the DC is often damaged during the swing phase of running. Weakness of the hamstring muscles was predicted to underlie this damage ${ }^{9-11)}$, and hamstring strengthening exercises have been proposed as a precaution ${ }^{12,13)}$. Also, the increase in stiffness of the hamstrings may serve as a cause of shoulder and elbow pain ${ }^{14)}$ and decrease of dynamic postural control ${ }^{15)}$. Although there are many reports on the injury of the DC and on the treatment of stiffness of the hamstrings ${ }^{16,17)}$, few studies have considered the difference between the PC and DC.

In the context of our clinical rehabilitation for patients who have undergone total knee arthroplasty, it was observed that flexibility of the DC of the ST is lost, but not that of the PC. Therefore, mechanical massage was often done in the popliteal regions ${ }^{18)}$. It was also observed that muscle force or range of motion in the knee joint increases with improved flexibility of the DC. However, this is difficult to prove clinically because the ST has a complex form.

Therefore, it is important to determine the functional differences between the PC and DC of the ST. In this study, using an ultrasound device, muscle fiber lengths in each compartment of the ST were measured during knee flexion and hip extension to determine the functional differences between the DC and PC as well as changes in joint angle.

\section{SUBJECTS AND METHODS}

The left legs of seven men aged 21-38 years, without orthopedic or neurological system disorders were examined. After the purpose of this study was explained to them, all of them provided written informed consent in order to participate. All procedures in this study were approved by our institutional ethics committee (No. 39-2).

The participants performed knee flexion (KF) and hip extension (HE) movements in the prone position, with $0^{\circ}$ extension of the knee joint and $25^{\circ}$ flexion of the hip joint. A band was fixed on the lumbar region and pelvis during movements. KF was defined as a flexion movement from about $0^{\circ}$ to $80^{\circ}$ knee flexion at $25^{\circ}$ hip flexion. HE was defined as an extension movement from about $25^{\circ}$ to $0^{\circ}$ hip flexion at $0^{\circ}$ knee flexion. These movement angles were selected with reference to those that occur during normal walking and because of the ease of the movements. Participants performed one movement every 4 seconds with the aid of an electric metronome. The TI of the ST was recorded at least twice, and the pennate part was recorded at least three times (Fig. 2a; see below for further details). The participants were allowed to rest between experiments. Before recording, the participants practiced the movements in order to maintain a fairly constant velocity and to avoid compensation caused by extension of the lumbar vertebrae during hip extension.

The images of the TI and pennate parts of the ST were captured using an ultrasound device (SSA-770A; Toshiba Medical Systems Corporation, Otawara, Tochigi, Japan). Shift of the TI position was measured from images of the TI, while changes in distal muscle fiber length, longitudinal length of the DC muscle, pennate angle, and muscle thickness were measured from images of the pennate part. Ultrasound images were converted to digital images at 60 frames per second by a personal computer. A probe with a linear form was used, taking care not to squeeze the muscle shape while lightly positioning the probe on the back of the thigh. One experimenter recorded all images, and two experimenters confirmed all images.

An ultrasound image of the TI is shown in Fig. 3a. Muscle length in the PC was appreciated from the shift of the intersection of the TI and superficial fascia (point A in Fig. 3a and 3c). It was inferred that the muscle length of the PC shortened when point A moved in a proximal direction and elongated when point A moved in a distal direction. This was because the part proximal to point A comprised muscle fibers of the PC. Sometimes, the shift in TI position was longer than the width of the probe, and it was unable to be measured. Therefore, sonic wave opacity tapes were affixed on the skin, and these tape points were considered a criteria point during repeated recordings.

An ultrasound image of the pennate part is shown in Fig. 3b. The pennate part was captured at the clearest point (which is usually located $65 \%$ toward the tibial tuberosity from the ischial tuberosity; Fig. 1). It was supposed that superficial fascia and muscle fibers that did not appear were in a straight line when the pennate part was measured (dotted line in Fig. 4). The length of muscle fiber in the DC and the longitudinal length of the DC muscle were calculated from the pennate angle and muscle 


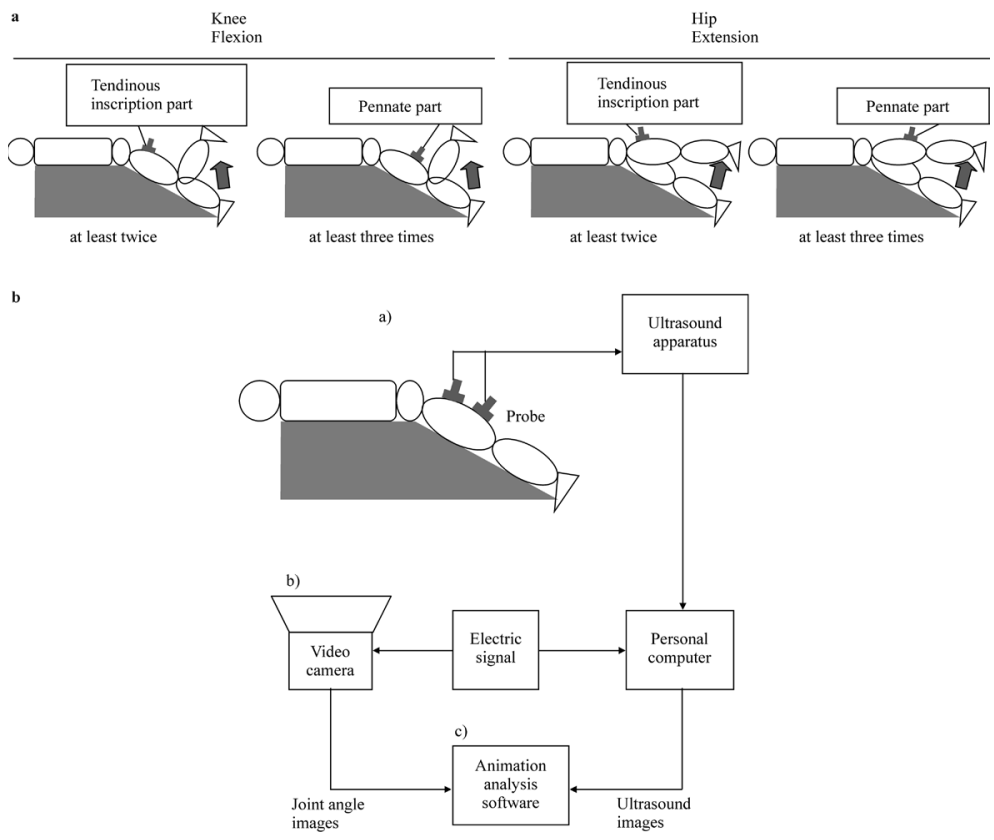

Fig. 2. Experimental set-up and methods of measurement

a. There were two movements and two recorded parts. In each movement (knee flexion and hip extension), the tendinous inscription and pennate parts were recorded at different times.

b. Ultrasound images were recorded at the tendinous inscription part and distal pennate part, during two movements, for each subject (a). These images were uploaded to a personal computer from an ultrasound device. The knee and hip joints angles were recorded using a video camera, in synchrony with the ultrasound images (c). The ultrasound (a) and joint angle (b) images were analyzed using DARTFISH animation software (c).
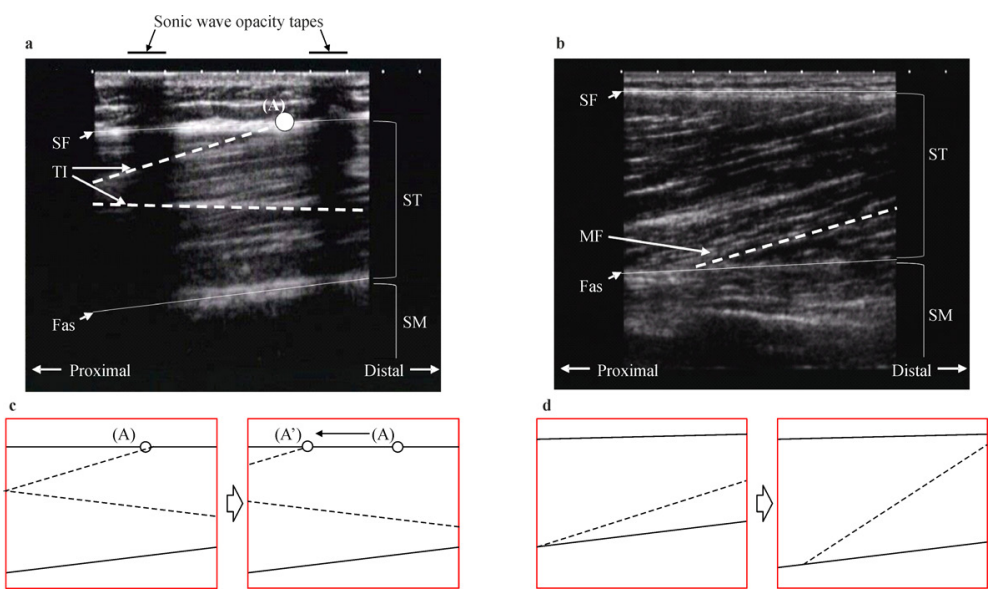

Fig. 3. Ultrasound images

(a) An example of the tendinous inscription part (see arrow 1 in Fig. 1). The tendinous inscription is V-shaped. Point (A) is the intersection of the tendinous inscription and superficial fascia. The muscle fiber on the left side of point $(\mathrm{A})$ represents the proximal compartment. Movement of point (A) is considered as a change in the muscle fiber length of the proximal compartment, as shown in c.

(b) An example of the pennate part of the distal compartment is depicted (see arrow 2 in Fig. 1). The muscle on the left side is thicker than distal right side. We recorded the same muscle fiber and thickness values in a single movement (see Fig. 4).

(c, d) Expressed models of the tendinous inscription part (c) and the pennate part (d) during movement of the knee and hip joints, respectively.

SF: superficial fascia; TI: tendinous inscription; Fas: fascia between the semitendinosus and semimembranosus muscles; ST: semitendinosus muscle; SM: semimembranosus muscle; MF: muscle fiber 


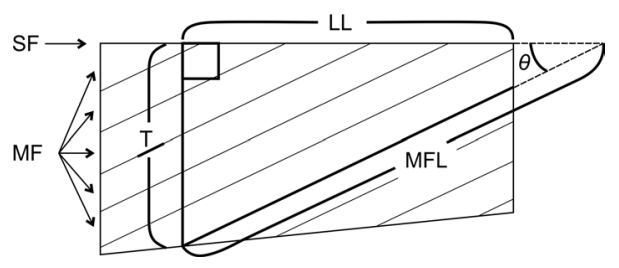

Fig. 4. Model of the pennate part of the semitendinosus muscle

The superficial fascia and muscle fiber are composed of two planes with pennate angle, $\theta$.

SF: superficial fascia; MF: muscle fiber; $\theta$ : pennate angle; LL: longitudinal length of the distal compartment muscles; MFL: muscle fiber length; T: muscle thickness

thickness, because the muscle fiber was longer than that shown by the ultrasound image (Fig. 4). Shortening and elongation of the muscle fiber length in the DC were determined relative to those during rest.

Joint movement was recorded by a video camera (GZ-HD300; Victor Company of Japan, Ltd., Yokohama, Kanagawa, Japan) during all experiments, simultaneously with ultrasound images. The images were recorded at 60 frames per second. The joint angles were measured from these images.

Joint angles were analyzed from video images and shift of the TI position, while muscle length of the DC, longitudinal length of the DC muscle, pennate angle of the DC, and muscle thickness of the DC were analyzed from ultrasound images. The averages of the clearer two of all recorded images and were used. The joint angle was measured using the automatic tracking mode of Dartfish (Dartfish Japan Co., Ltd., Shibuya-ku, Tokyo, Japan). Each time the joint angles changed by $1^{\circ}$, the other data were measured manually because ultrasound images were too unclear to use an automatic mode.

Four data points for decreases in muscle length were obtained for each participant. These were measured in both the TI and pennate parts during KF and $\mathrm{HE}$ (one point for each part during each movement). In addition, the sum of the decreases in muscle length of the PC muscles and the longitudinal length of the DC muscles were obtained during KF and HE. The measurement errors, errors arising owing to unclear images, and analysis errors for the DC were greater than those for the PC, because the length of the DC muscle fibers was calculated using two measurements (i.e., pennate angle and muscle thickness). Thus, three data points were obtained for the pennate part in order to calculate the average of every three measurements and to decrease the effects of these errors.

Statistical computing R (version 3.0.1; The R Foundation for Statistical Computing, Vienna, Austria) was used for statistical analysis. Decreases in muscle fiber length and the joint angle in both compartments were correlated using the Pearson's correlation coefficient. Significance was set at $\mathrm{p}<0.05$.

\section{RESULTS}

During KF, there were significant correlations in both compartments, with decreases in muscle fiber length and joint angle in both compartments showing correlations ( $\mathrm{PC}, \mathrm{r}=0.75, \mathrm{p}<0.00001 ; \mathrm{DC}, \mathrm{r}=0.54, \mathrm{p}<0.00001)$. During HE, there were no significant correlations in either compartment $(\mathrm{PC} ; \mathrm{r}=-0.12, \mathrm{p}=0.134, \mathrm{DC} ; \mathrm{r}=-0.13, \mathrm{p}=0.111)$. There were significant correlations in longitudinal length of the whole $\mathrm{ST}$ and joint angle during $\mathrm{KF}$ and $\mathrm{HE}(\mathrm{KF}, \mathrm{r}=0.86, \mathrm{p}<0.00001 ; \mathrm{HE}, \mathrm{r}=0.19, \mathrm{p}=0.037)$.

The decreases in muscle fiber length and joint angle are presented in Fig. 5. During KF in both compartments (Fig. 5a and 5c), when the knee flexion angle increased, muscle fiber length decreased. However, during HE in both compartments, the decreases in muscle fiber length were disconnected (Fig. 5c and 5d).

Only the decreases in muscle fiber length in the DC during HE tended to be negative; in other words, the muscle fibers were extended (Fig. 5d). The other decreases in muscle fiber length tended to be positive; in other words, the muscle fibers shortened.

The mean and standard deviation of the changes in pennate angle and muscle thickness in the DC are shown in Table 1. Overall, the means increased.

\section{DISCUSSION}

This study aimed to identify the functional differences between the PC and DC of the ST during KF or HE. Decreases in muscle fiber length in the PC and DC were measured using ultrasound exam, and the correlation between decreases in muscle fiber length and joint angle were calculated. Previous studies have reported differences in the examined muscles. These studies compared muscle activity in the medial and lateral sites in the biceps brachii longus muscle using wire electrodes and reported a difference in the activities of these muscles during elbow flexion, forearm supination, or shoulder exorotation $^{19,20)}$. Additionally, some studies compared muscle fiber and tendon length during walking using an ultrasound device and reported differences in the length of these fibers during each walking phase ${ }^{21,22)}$. However, the aforementioned studies did not examine in-series muscle fibers as well as the PC and DC of ST. The present study hypothesized that the proximal 

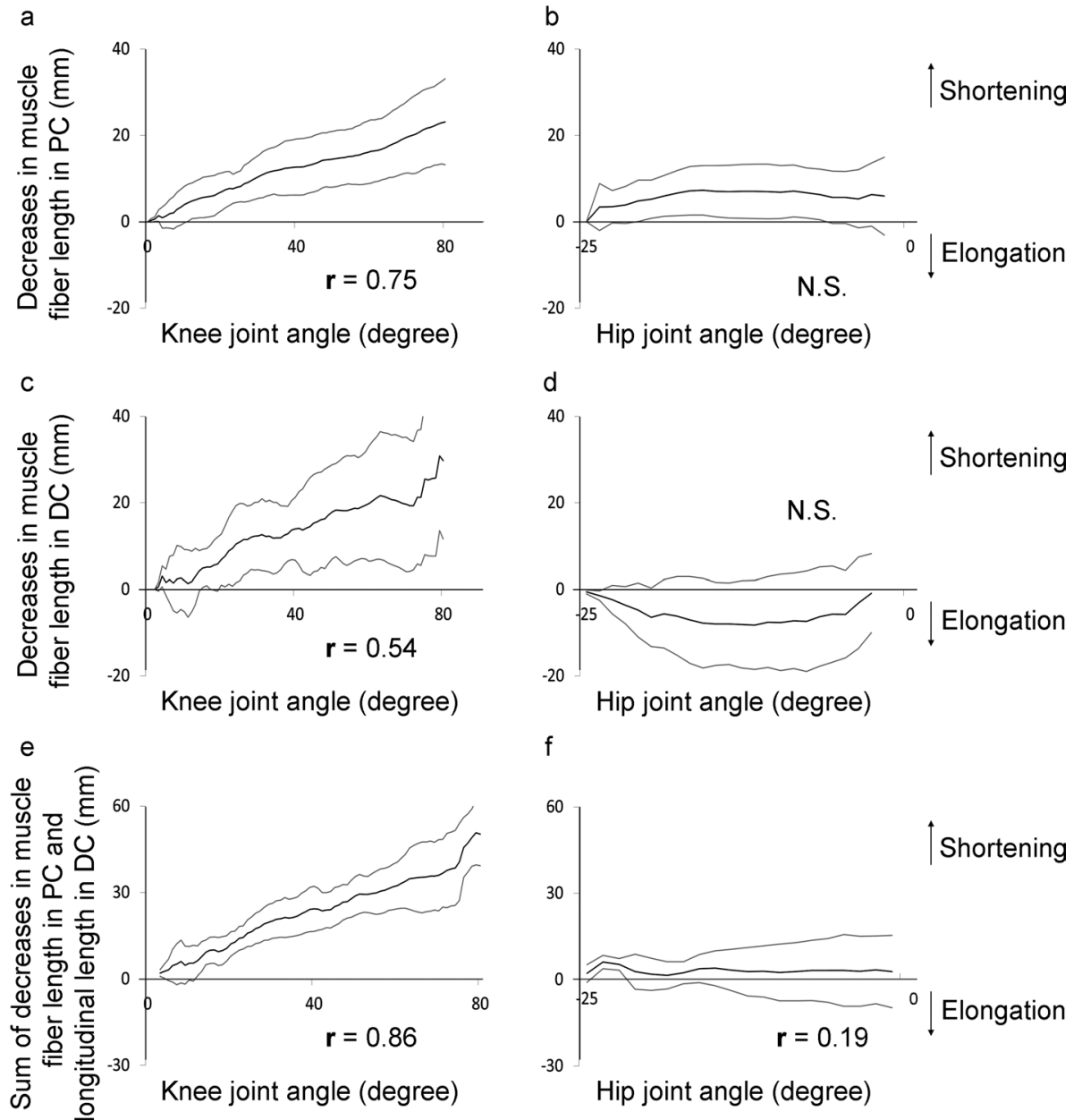

f

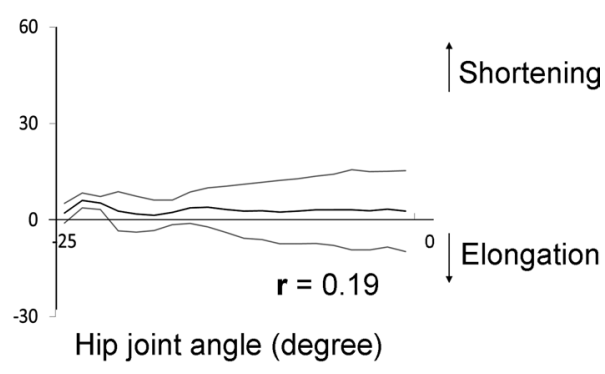

Fig. 5. Decreases in muscle fiber length during joint movement

The graphs depict decreases in muscle fiber length in (a) the proximal compartment (PC) during knee flexion; (b) the proximal compartment during hip extension; (c) the distal compartment (DC) during knee flexion; (d) the distal compartment during hip extension; and decreases in the sum of muscle fiber length of the proximal compartment and longitudinal length of the distal compartment (e) during knee flexion and (f) during hip extension. r, Pearson's correlation coefficient. N.S., not significant. The black lines indicate the mean of the changes in muscle fiber length. The gray lines indicate the standard deviation of the decreases in muscle fiber length. There was a strong correlation between joint angle and muscle fiber length during knee flexion ( $\mathrm{a}$ and $\mathrm{b}$ ). During hip extension (c and d), there was no correlation between joint angle and muscle fiber length.

Table 1. Changes in pennate angle of the distal compartments and muscle thickness of the distal compartments for all subjects

\begin{tabular}{lcccc}
\hline & \multicolumn{2}{c}{ Knee flexion } & \multicolumn{2}{c}{ Hip extension } \\
\cline { 2 - 5 } & $\begin{array}{c}\text { Pennate angle } \\
(\text { degrees })\end{array}$ & $\begin{array}{c}\text { Muscle thickness } \\
(\mathrm{cm})\end{array}$ & $\begin{array}{c}\text { Pennate angle } \\
(\text { degrees })\end{array}$ & $\begin{array}{c}\text { Muscle thickness } \\
(\mathrm{cm})\end{array}$ \\
\hline Subject 1 & $4.6 \pm 3.4$ & $-0.7 \pm 3.1$ & $-2.1 \pm 2.2$ & $2.2 \pm 2.3$ \\
Subject 2 & $3.7 \pm 2.0$ & $0.9 \pm 1.3$ & $4.2 \pm 4.0$ & $5.1 \pm 5.2$ \\
Subject 3 & $6.6 \pm 4.7$ & $1.3 \pm 2.0$ & $2.1 \pm 1.9$ & $2.9 \pm 1.9$ \\
Subject 4 & $4.8 \pm 3.3$ & $3.1 \pm 1.5$ & $1.7 \pm 2.0$ & $4.4 \pm 2.1$ \\
Subject 5 & $9.4 \pm 4.2$ & $3.2 \pm 3.1$ & $4.7 \pm 3.8$ & $4.0 \pm 2.6$ \\
Subject 6 & $5.0 \pm 2.4$ & $1.1 \pm 1.2$ & $4.2 \pm 2.4$ & $4.5 \pm 2.2$ \\
Subject 7 & $5.0 \pm 3.5$ & $-0.1 \pm 1.9$ & $0.7 \pm 3.8$ & $4.9 \pm 2.8$ \\
\hline
\end{tabular}

During knee flexion, the pennate angle increased and muscle thickness tended to increase. During hip extension, the pennate angle tended to increase and muscle thickness increased.

Values are shown as mean \pm standard deviation. 
muscle fiber and distal muscle fiber might have functional differences.

It was found that decreases in muscle fiber length in the PC and DC correlated with the joint angle during KF. In addition, the longitudinal length of the whole ST was correlated with the joint angle during KF. The difference in correlation was too small to determine the exact functional difference between the $\mathrm{PC}$ and $\mathrm{DC}$, and it is possible that there are not functional differences between the PC and DC during KF.

During HE, there were no significant correlations between decreases in muscle fiber length in the PC and DC and joint angles. However, there were differences in the shortening or elongation of muscle fibers; i.e., these displayed different fascicle behavior. These results differed from other results in that the muscle fiber of the PC shortened during HE. The pennate muscle can control the change of muscle tendon complex length by changing the pennate angle ${ }^{23)}$. This difference during HE could be meaningful and was one of the functional differences.

It is important to note that the measurement methods used for each compartment were different. Measurement of decreases in muscle fiber length in the PC used the shift of the TI, recording all muscle fibers of the PC. Conversely, measurement of the DC did not cover all muscle fibers of the DC, but only those in the pennate part. Also, it is necessary to obtain threedimensional measurements because the ST is staggered ${ }^{5)}$, and it is possible that this staggering differs between the PC and DC. Next, it is necessary to consider that each compartment affects the tension of the other. When one compartment is undergoing contraction, afferent information from muscle spindles and Golgi tendon organs in the other compartment changes. The response from afferent information was different even in the same muscle at every site, e.g. medial and lateral head of the gastrocnemius ${ }^{24)}$. In ST muscle, the afferent information of PC and DC may produce different responses. During KF, the correlation between the PC and DC did not determine those differences, which suggested that the PC and DC produced similar level of tension. During HE, the muscle fiber of the DC might be eccentrically contracted, although it might be passively stretched by muscle activity of the PC. Muscle activity increases the pennate angle and muscle thickness. These both increased in the DC during HE. The muscle activity of the DC might be weaker than that of the PC, but would not be inactive.

In addition, the longitudinal length of the whole ST was correlated with joint angle during HE. The fascicle behavior during HE differed between the PC and DC, but both compartments shortened as a whole because the muscle activity was coordinated. It was unclear why the PC, parallel muscle, contracted stronger than did the DC during HE. In parallel muscles, the shortening velocity is faster and the range of motion is larger than those in pennate muscles. During HE, the change of muscle tendon complex length should be smaller, because few joint ranges were measured. During HE, it was predicted that the PC played fewer roles and contracted weakly, but our results did not support this prediction. The reason might be the effect of the slow velocity motion. Slow velocity motion does not require strong contraction. Therefore, the DC that exerted stronger force might contract more weakly than does the PC.

Our results can be applied in clinical contexts. In the context of sports, it is thought that the ST is prone to injury ${ }^{7)}$, particularly during eccentric contraction ${ }^{3,8)}$. Our results suggest that muscle fibers tend to elongate in the DC, which may be the cause of many injuries to the DC of the ST. In addition, rapid hip extension may cause injury to the DC of the ST, because hip extension caused elongation of the muscle fibers of the DC.

There are three main limitations of this study. First, the muscle fiber length of the PC was measured movement for the skin, but movement of the skin for the bone was not measured. The skin around the joint moved to a greater extent for the bone during joint movement ${ }^{25}$, which may have caused a skin effect. Second, muscle activity was not measured. The proportion of muscle activity that each compartment contributed to a movement could not be determined and the muscle activity was not measured with, for example, electromyograms. In addition to the gluteus maximus, the gluteus medius, gluteus minimus, hamstrings, and adductors muscles act as hip extensors. Additionally, the hamstrings and gracilis, sartorius, popliteus, and gastrocnemius muscles act as knee flexors. Regarding the hip joint in particular, the contribution of the ST may be minimal, because the correlation of the change in joint angle and decreases in muscle fiber length was not significant.

Finally, a single joint movement was examined by one method of force production and contraction velocity through a partial range of its motion. These components of movement were important with respect to the differences between parallel and pennate muscles. In addition, during walking and other activities, there are other effects, such as ground force reaction, and the activity of other muscles. The clinical implications of our findings may be limited, and it is necessary to perform additional investigation and to consider various other factors.

\section{ACKNOWLEDGEMENTS}

We thank Mr. Segawa for technical assistance with the ultrasound apparatus and Mr. Sone for insightful comments and suggestions, and are grateful to everyone concerned with this study.

\section{REFERENCES}

1) Kandel ER, Schwartz JH, Jessell TM, et al.: Principles of neural science, 5th ed. New York: McGraw Hill Companies, 2013, pp 768-789.

2) Kawakami Y: Undotyu no kinseni shuushuku doutai. J Soc Biomech, 2003, 27: 67-71 (in Japanese). [CrossRef]

3) Azizi E, Roberts TJ: Geared up to stretch: pennate muscle behavior during active lengthening. J Exp Biol, 2014, 217: 376-381. [Medline] [CrossRef] 
4) Ward SR, Eng CM, Smallwood LH, et al.: Are current measurements of lower extremity muscle architecture accurate? Clin Orthop Relat Res, 2009, 467: 1074-1082. [Medline] [CrossRef]

5) Woodley SJ, Mercer SR: Hamstring muscles: architecture and innervation. Cells Tissues Organs, 2005, 179: 125-141. [Medline] [CrossRef]

6) An XC, Lee JH, Im S, et al.: Anatomic localization of motor entry points and intramuscular nerve endings in the hamstring muscles. Surg Radiol Anat, 2010, 32: 529-537. [Medline] [CrossRef]

7) Koulouris G, Connell D: Evaluation of the hamstring muscle complex following acute injury. Skeletal Radiol, 2003, 32: 582-589. [Medline] [CrossRef]

8) Proske U, Morgan DL, Brockett CL, et al.: Identifying athletes at risk of hamstring strains and how to protect them. Clin Exp Pharmacol Physiol, 2004, 31: 546-550. [Medline] [CrossRef]

9) Lieber RL, Woodburn TM, Fridén J: Muscle damage induced by eccentric contractions of 25\% strain. J Appl Physiol 1985, 1991, 70: 2498-2507. [Medline]

10) Stanton P, Purdham C: Hamstring injuries in sprinting — the role of eccentric exercise. J Orthop Sports Phys Ther, 1989, 10: 343-349. [Medline] [CrossRef]

11) Kumazaki T, Ehara Y, Sakai T: Anatomy and physiology of hamstring injury. Int J Sports Med, 2012, 33: 950-954. [Medline] [CrossRef]

12) Croisier JL, Forthomme B, Namurois MH, et al.: Hamstring muscle strain recurrence and strength performance disorders. Am J Sports Med, 2002, 30: 199-203. [Medline]

13) Croisier JL, Ganteaume S, Binet J, et al.: Strength imbalances and prevention of hamstring injury in professional soccer players: a prospective study. Am J Sports Med, 2008, 36: 1469-1475. [Medline] [CrossRef]

14) Endo Y, Sakamoto M: Correlation of shoulder and elbow injuries with muscle tightness, core stability, and balance by longitudinal measurements in junior high school baseball players. J Phys Ther Sci, 2014, 26: 689-693. [Medline] [CrossRef]

15) Endo Y, Sakamoto M: Relationship between lower extremity tightness and star excursion balance test performance in junior high school baseball players. J Phys Ther Sci, 2014, 26: 661-663. [Medline] [CrossRef]

16) Nishikawa Y, Aizawa J, Kanemura N, et al.: Immediate effect of passive and active stretching on hamstrings flexibility: a single-blinded randomized control trial. J Phys Ther Sci, 2015, 27: 3167-3170. [Medline] [CrossRef]

17) Cho SH, Kim SH, Park DJ: The comparison of the immediate effects of application of the suboccipital muscle inhibition and self-myofascial release techniques in the suboccipital region on short hamstring. J Phys Ther Sci, 2015, 27: 195-197. [Medline] [CrossRef]

18) Kim SM, Kim SR, Lee YK, et al.: The effect of mechanical massage on early outcome after total knee arthroplasty: a pilot study. J Phys Ther Sci, 2015, 27: 3413-3416. [Medline] [CrossRef]

19) ter Haar Romeny BM, van der Gon JJ, Gielen CC: Relation between location of a motor unit in the human biceps brachii and its critical firing levels for different tasks. Exp Neurol, 1984, 85: 631-650. [Medline] [CrossRef]

20) Denier van der Gon JJ, ter Haar Romeny BM, van Zuylen EJ: Behaviour of motor units of human arm muscles: differences between slow isometric contraction and relaxation. J Physiol, 1985, 359: 107-118. [Medline] [CrossRef]

21) Fukunaga T, Kubo K, Kawakami Y, et al.: In vivo behaviour of human muscle tendon during walking. Proc Biol Sci, 2001, 268: 229-233. [Medline] [CrossRef]

22) Mian OS, Thom JM, Ardigò LP, et al.: Gastrocnemius muscle-tendon behaviour during walking in young and older adults. Acta Physiol (Oxf), 2007, 189: 57-65. [Medline] [CrossRef]

23) Brainerd EL, Azizi E: Muscle fiber angle, segment bulging and architectural gear ratio in segmented musculature. J Exp Biol, 2005, 208: 3249-3261. [Medline] [CrossRef]

24) Scott JG, Mendell LM: Individual EPSPs produced by single triceps surae Ia afferent fibers in homonymous and heteronymous motoneurons. J Neurophysiol, 1976, 39: 679-692. [Medline]

25) Fukui T, Otake Y, Kondo T: In which direction does skin move during joint movement? Skin Res Technol, 2015, [Epub ahead of print] [CrossRef]. [Medline] 